\title{
Thin-for-gestational age infants are at increased risk of neurodevelopmental delay at 2 years
}

\author{
Sinéad M O'Neill, ${ }^{1}$ Geraldine Hannon, ${ }^{2}$ Ali S Khashan, ${ }^{1,3} \mathrm{~J}$ O'B Hourihane, ${ }^{2}$ \\ Louise C Kenny, ${ }^{1,4}$ Mairead Kiely, ${ }^{1,5}$ Deirdre M Murray ${ }^{1,2}$
}

\begin{abstract}
- Additional material is published online only. To view please visit the journal online (http://dx.doi.org/10.1136/ archdischild-2016-310791).

For numbered affiliations see end of article.
\end{abstract}

\section{Correspondence to} Dr Deirdre M Murray, Neonatal Brain Research Group, Cork University Maternity Hospital, Wilton T12 DC4A, Cork, Ireland; D.Murray@ucc.ie

Received 10 March 2016 Revised 30 August 2016 Accepted 30 September 2016 Published Online First 20 October 2016

\section{Linked}

- http://dx.doi.org/10.1136/ archdischild-2016-312103

\section{CrossMark}

To cite: O'Neill SM, Hannon G, Khashan AS, et al. Arch Dis Child Fetal Neonatal Ed 2017;102:F197-F202.

\section{ABSTRACT}

Background Infants born small-for-gestational age

(SGA) are at increased risk of developmental difficulties. Identifying those most at risk is challenging. We examined the effect of neonatal body composition and customised birthweight centiles on neurocognitive and behavioural outcomes at age 2 .

Study design Prospective cohort study of term infants from the Cork BASELINE Birth Cohort Study classified into the following exposure groups: a birth weight $<10$ th customised centile (SGA, n=51); body fat percentage at birth $<10$ th centile (thin-for-gestational age (TGA, $n=51))$ or both SGA and TGA infants (small- and thin-for-gestational age (STGA), $n=13$ ). The SGA, TGA and STGA groups were compared with a reference (unexposed) group of appropriate-forgestational age (AGA, $n=189)$ infants. Outcome was assessed at 24 months using the Bayley Scales of Infant Development Version III and the Child Behaviour Checklist.

Results Outcomes in the SGA infants did not differ significantly from the AGA group. TGA infants had significantly lower scores across all three domains, with a $0.35,0.38$ and 0.41 SD reduction in language, cognitive and motor scale scores, respectively. STGA infants had poorer cognitive outcome with a median cognitive scale score of 90 (IQR 85-95) compared with 95 (IQR 90-100) in the AGA reference group, $p=0.005$. The adjusted OR of developmental delay at 2 years was $5.00(95 \% \mathrm{Cl} 1.46$ to $17.13, \mathrm{p}=0.010)$ in the STGA group.

Conclusion TGA infants, in particular those born STGA, are at increased risk of developmental delay at 2 years compared with the AGA infants.

\section{BACKGROUND}

Long-term neurodevelopmental disability arising from perinatal events is a major global economic burden. ${ }^{1}{ }^{2}$ There is increasing evidence that the intrauterine environment plays a significant role in fetal growth and on the long-term health of the child. ${ }^{3}$ Fetal growth restriction (FGR) refers to a fetus that does not reach its genetically predetermined growth potential and may be caused by many factors, most commonly placental insufficiency. ${ }^{45}$ FGR also affects the growth and development of the brain with changes in brain weight, structure, myelination and connectivity. ${ }^{6}$ FGR is suggested in the antenatal period by ultrasound assessment of slowing growth trajectories and abnormal Doppler indices of umbilical and middle cerebral arterial flow and pulsatility. ${ }^{7}$ Ultrasound estimation of fetal weight suffers from imprecision ${ }^{8}$

\section{What is already known on this topic?}

Being small-for-gestational age at birth is a risk factor for later cognitive and behavioural difficulties.

- Using birth weight or birthweight centiles alone are not reliable indicators of risk as many children may be small but normal.

\section{What this study adds?}

Being thin-for-gestational age (percentage body fat $<10$ th percentile) is also a risk factor for developmental delay at 2 years.

- The greatest risk was seen in those children who both were small and thin at birth.

- Measurement of body composition at birth in small-for-gestational age infants may help to assign risk.

and is largely unavailable in low resource settings. Consequently, birth weight is frequently used as a surrogate measure. Infants with birth weight lower than the 10th percentile are termed small-for-gestational age (SGA). ${ }^{9}$ Infants who are born SGA have a greater likelihood of long-term cognitive impairment, lower social competency and behavioural problems. ${ }^{10-13}$ Most research in this area has been carried out in preterm SGA infants, where early gestation and low birth weight combine to worsen outlook. ${ }^{1} 1415$

In term infants, identifying those at greatest risk is more difficult. Up to one-third of SGA infants are not FGR but are simply constitutionally small, ${ }^{16}$ and while identification of FGR infants is improved by the use of birthweight centiles, their use alone will still include many normal infants. ${ }^{17}$ These difficulties have led to inconsistent findings, with some studies showing a modest or negligible effect of SGA on cognitive outcome when standard centiles alone are used. ${ }^{18}$ Improved postnatal assessment of risk would help clinicians and parents to target follow-up and early intervention. Individualised or customised birth centiles, which adjust for maternal ethnicity, maternal or biparental height and fetal birth order, are now available and have been shown to detect FGR in stillborn infants who would otherwise have been classified as appropriate-for-gestational age (AGA). ${ }^{19} \quad{ }^{20} \quad$ In 
recent years, the ability to accurately measure body composition using air displacement body plethysmography in the newborn period has provided additional information on the influence of the in utero environment on fetal growth. We have previously reported normative reference ranges for body composition at birth in term neonates. ${ }^{21}$ We wished to examine whether early body composition measurement could more accurately identify infants at increased risk of long-term neurocognitive and behavioural deficits compared with customised birthweight centiles alone.

\section{METHODS}

Study design and group selection

Participants were term infants (gestational age $\geq 37$ weeks) recruited from the Cork BASELINE Birth Cohort Study born between March 2009 and September 2011. BASELINE (http:/ www.baselinestudy.net) was established in 2008 as a follow-up to the Screening for Pregnancy Endpoints (SCOPE) pregnancy study. The study participants, aims and methods of the Cork BASELINE birth cohort study have been previously reported. ${ }^{22}$ Body fat estimation of the cohort began in March 2009. The SCOPE study (http://www.scopestudy.net/) is a worldwide multicentre study involving primiparous low-risk women aimed at establishing biomarkers to assist with prediction and prevention of the major diseases of late pregnancy. ${ }^{23}$ Women in the SCOPE study were recruited antenatally at 15 weeks' gestation to the BASELINE study. Ethical approval for the Cork BASELINE Birth Cohort Study was granted by the Clinical Research Ethics Committee of the Cork Teaching Hospitals (Ref: ECM 5 (9) 01/ 07/2008), and the study is registered (Ref: NCT01498965) with the United States National Institutes of Health Clinical Trials Registry (http://www.clinicaltrials.gov).

A prospective cohort study design was used. The study sample was selected using the following criteria to select the exposed groups: (1) SGA infants defined as a customised birthweight centile $<10$ th centile; (2) thin-for-gestational age (TGA) infants defined as those having a body fat percentage $(\% \mathrm{BF})$ at 2 days $<10$ th centile for gestation and sex and (3) both smalland thin-for-gestational age (STGA) infants. SGA, TGA and STGA infants are defined as the exposed groups. AGA infants (reference/unexposed group) comprised a computer-generated stratified random sample selected from across the customised birth centiles (CBCs); first quartile between 10th and 25th $\mathrm{CBC}$, second quartile between 25 th and 50th CBC, third quartile from 50th to 75 th $\mathrm{CBC}$ and the fourth quartile from the 75 th to 100th CBC using the statistical software Stata (V.14; StataCorp, College Station, Texas, USA). AGA infants also had a $\% \mathrm{BF}>10$ th centile. Overlap existed between the SGA and TGA groups, and so those infants who were both STGA were nominated for post hoc analysis.

\section{Sample size calculations}

To ensure that the study would have sufficient power to answer the question posed, the authors wished to be able to detect a medium effect size (0.30 Cohen's D effect) or a difference in 5-6 IQ points. A mean (SD) cognitive score of 100 (15) for the reference population was estimated. This would require an estimated starting population of over 1000 infants with body composition measurement at birth. The authors aimed to select exposed and unexposed infants in a 1:2 ratio and to have $\mathrm{n}=110$ SGA, $\mathrm{n}=110$ TGA and $\mathrm{n}=220$ reference infants giving a total nested group of 440 infants. In fact, the number of infants with a customised birth weight $<10$ th percentile was less than expected $(n=80)$ (the reason for this is that the eligibility criteria for the SCOPE study included all low-risk primiparous women, who in the study area of Cork, Ireland, were largely derived from an upper socioeconomic (SE) strata and hence lower than anticipated rates of FGR) and the number with body fat $<10$ th percentile was $n=81$. The remaining infants formed the reference (unexposed) sample $(n=299)$.

\section{Anthropometry}

Naked weight was measured at birth using digital scales (seca 384; seca, Birmingham, UK) correct to the nearest $0.1 \mathrm{~kg}$. Supine length correct to the nearest $0.1 \mathrm{~cm}$ was measured at birth (seca 210). SGA was defined as a birth weight $<10$ th using individualised customised percentiles adjusted for maternal height, booking weight and ethnicity and for infant gestational age and sex. ${ }^{20}$ Fat mass $(\mathrm{FM})$ and fat-free mass $(\mathrm{kg})$ were measured within 4 days of birth using the PEA POD Infant Body Composition Tracking System (COSMED USA, Concord, California, USA). The PEA POD uses air displacement plethysmography to accurately measure body composition in infants weighing between 1 and $8 \mathrm{~kg}$ and has shown high levels of agreement with the gold standard four-compartment model. Body fat percentage $(\% \mathrm{BF})$ was calculated as $[(\mathrm{FM}(\mathrm{kg}) / \mathrm{body}$ mass $(\mathrm{kg})) \times 100]$. TGA was defined as a $\% \mathrm{BF}<10$ th centile based on previously published reference ranges for the cohort. ${ }^{21}$

\section{Two-year outcome assessment}

Participants identified as eligible at birth were approached at their 2-year follow-up assessment and asked to return for an additional visit for a more detailed neurodevelopmental assessment. Outcome was assessed at 24 months using the Bayley Scales of Infant and Toddler Development Version III (BSID-III). ${ }^{24}$ This was administered to all in children studied by a single research psychologist $(\mathrm{GH})$ trained in administration and scoring of the BSID-III and blinded to the anthropometric data or exposed/unexposed status of the participants. The assessment consists of five scales: cognition, receptive communication, expressive communication, fine motor and gross motor, with receptive and expressive communication combined to form a language composite score and fine and gross motor combined to form a motor composite score. Published normative scores for the composite scales of the BSID-III are a mean of 100 with a SD of 15; an abnormal BSIDIII composite scale score was defined as $<85$ in any of the three measured domains: cognitive, motor or language. BSID-III scores of $<85$ are recognised as identifying children in the low average range for development. The 2006 restandardised BSID-III has been shown to underestimate developmental delay compared with the Bayley II version. The cut-off value of $<85$ for cognitive and language has been shown recently to give the best prediction of moderate-to-severe developmental delay. ${ }^{25}$ Families unable to attend the research centre were offered the option of their child completing the test in their own home. The age range limits set for collecting data on outcome measures were from 24 months 0 days to 30 months 29 days.

At the 24-month assessment, emotional and behavioural problems in all participants were assessed by the Child Behaviour Checklist for 1.5-5 years (CBCL/1.5-5); a 99-item checklist completed by parents/caregivers, indicating the frequency of particular behaviours over the past 2 months on a three-point scale. $^{26}$ The CBCL/1.5-5 comprises two broadband scales, internal problem score (IPS) and external problem score (EPS). The IPS is made up of scores from four individual syndrome scales: emotionally reactive, anxious/depressed, somatic complaints and withdrawn. The EPS is made up of scores from two 
individual syndrome scales: attention problems and aggressive behaviour. A total problem score (TPS) is calculated to give an overall view of the child's behaviour. For all scales, scores at the 93rd percentile or above were designated as borderline and scores at the 98th percentile or above as clinical. For this analysis, scores in the borderline and clinical range ( $>93 \mathrm{rd}$ percentile) are denoted as clinical range. This equates to $>14$ for IPS, $>21$ for EPS and $>52$ for TPS.

\section{Statistical analysis}

Descriptive statistics were generated for each group (SGA, TGA, STGA and the AGA reference group). Continuous data were examined for normality by skewness/kurtosis. As BSIDIII data were negatively skewed for some subscales, values are presented as median with IQR. Differences between the groups were examined individually as SGA versus AGA, TGA versus AGA and STGA versus AGA using the Mann-Whitney $U$ test for non-parametric data and Student's t-test for approximately normally distributed data. Effect size on overall group scores was calculated using Hedges' $g$ effect size calculation ${ }^{27}$ due to the different sample sizes in the comparison groups. Statistical significance was set at $\mathrm{p}<0.05$. Univariate and multivariateadjusted logistic regression models were generated to estimate the effect of the exposure (ie, being SGA, TGA and STGA) compared with the AGA reference category in one model on neurodevelopmental outcomes with a score of $<85$ used as the cut-off for each binary outcome. The multivariate logistic regression was adjusted for maternal characteristics including maternal age, smoking status (self-reported), marital status, maternal years of schooling and SE index (which was measured using the New Zealand Socio-Economic Index of Occupational Status). ${ }^{28}$ Results are presented as crude and adjusted ORs with 95\% CIs generated using the statistical software package IBM SPSS for Windows V.22.0 (Released 2013; IBM, Armonk, New York, USA).

\section{RESULTS}

\section{Baseline characteristics}

The total eligible cohort comprised 1942 term infants, of whom both customised birthweight centiles and body fat measurements at birth were available for 1107 infants. A further 23 infants were excluded as their \%BF was measured after 4 days of age, leaving a final cohort of 1084 infants. There were 80 SGA infants and 81 TGA infants in the entire cohort. Of note, 20 infants fulfilled both criteria and were termed STGA. From the total cohort of AGA infants, 299 infants were identified to provide the reference group. We aimed to have two reference infants in the AGA group for each exposed infant in the SGA and TGA groups to allow for loss to follow-up over the 2-year study period (see online supplementary figure S1).

SGA and TGA infants did not differ according to maternal characteristics including maternal age, education level or SE index (table 1). Both groups had higher rates of maternal smoking compared with the AGA group. The TGA group had a higher percentage of female infants.

\section{Neurodevelopmental outcome}

In all 239/299 AGA (78\%), 67/80 (84\%) SGA and 69/81 (85\%) TGA infants remained within the study and returned for detailed developmental assessment at 2 years. Infants remaining in the study and who were assessed developmentally were more likely to be born to older women (>30 years (61\% vs $40 \%$, $\mathrm{p}=0.0009)$ ), married $(74 \%$ vs $61 \%, \mathrm{p}=0.0146)$, women who did not smoke $(77 \%$ vs $70 \%, p=0.3963)$ and women with a

\begin{tabular}{|c|c|c|c|}
\hline $\begin{array}{l}\text { Infant and maternal } \\
\text { characteristics }\end{array}$ & $\begin{array}{l}\text { SGA } \\
(n=80)^{*}\end{array}$ & $\begin{array}{l}\text { TGA } \\
(n=81)^{*}\end{array}$ & AGA $(n=299)$ \\
\hline Gestational age (weeks) & $39.64 \pm 1.3$ & $39.53 \pm 1.4$ & $39.81 \pm 1.2$ \\
\hline $\mathrm{p}$ Value & 0.304 & 0.134 & \\
\hline Sex, male, n (\%) & $43 / 80(54 \%)$ & $26 / 81(32 \%)$ & $140 / 299(47 \%)$ \\
\hline $\mathrm{p}$ Value & 0.321 & 0.013 & \\
\hline Birth weight $(\mathrm{g})$ & $2850 \pm 255$ & $3215 \pm 394$ & $3567 \pm 422$ \\
\hline $\mathrm{p}$ Value & $<0.001$ & $<0.001$ & \\
\hline Customised birthweight centile & $5.3 \pm 2.7$ & $30.6 \pm 24.7$ & $53.2 \pm 27.0$ \\
\hline $\mathrm{p}$ Value & $<0.001$ & $<0.001$ & \\
\hline Length $(\mathrm{cm})$ & $48.4 \pm 1.7$ & $49.5 \pm 2.0$ & $50.6 \pm 1.9$ \\
\hline $\mathrm{p}$ Value & $<0.001$ & $<0.001$ & \\
\hline Body fat, $\mathrm{n}(\%)$ & $8.1(3.5)$ & $4.0(1.1)$ & $11.4(3.7)$ \\
\hline$p$ Value & $<0.001$ & $<0.001$ & \\
\hline Maternal age (years) & $30.0 \pm 4.8$ & $30.2 \pm 4.0$ & $29.8 \pm 4.4$ \\
\hline $\mathrm{p}$ Value & 0.974 & 0.813 & \\
\hline Maternal total years of schooling & $13.3 \pm 0.8$ & $13.3 \pm 1.0$ & $13.2 \pm 1.2$ \\
\hline$p$ Value & 0.476 & 0.531 & \\
\hline $\begin{array}{l}\text { Mother completed tertiary } \\
\text { education, } \mathrm{n}(\%)\end{array}$ & $39(49)$ & $45(55)$ & $168(56)$ \\
\hline $\mathrm{p}$ Value & 0.127 & 0.924 & \\
\hline Socioeconomic index & $42.36 \pm 17.3$ & $46.12 \pm 18.6$ & $42.51 \pm 15.6$ \\
\hline$p$ Value & 0.509 & 0.192 & \\
\hline Smoking, n (\%) & $14(17.5)$ & $11(13.6)$ & $23(7.7)$ \\
\hline$p$ Value & 0.005 & 0.018 & \\
\hline \multicolumn{4}{|c|}{$\begin{array}{l}\text { Data are given as } n(\%) \text { or mean } \pm S D \text {; a p value }<0.05 \text { denotes a statistically } \\
\text { significant difference compared with AGA group using the Mann-Whitney U test or } \chi^{2} \\
\text { test as appropriate. Socioeconomic index was measured using the New Zealand } \\
\text { socioeconomic index. } \\
\text { *SGA and TGA groups include } 20 \text { infants who were both SGA and TGA. } \\
\text { AGA, appropriate-for-gestational age; SGA, small-for-gestational age; STGA, small- } \\
\text { and thin-for-gestational age; TGA, thin-for-gestational age. }\end{array}$} \\
\hline
\end{tabular}

higher SE index $(88 \%$ vs $80 \%, \mathrm{p}=0.069)$, compared with those who were not assessed. Not all domains were completed in all children, and results are provided based on those children who were able to complete the assessment. On occasion this required more than one visit to complete all domains. The median (IQR) composite scores achieved in each of the study groups are displayed in table 2 .

\section{Outcome in SGA infants}

There were no differences in cognitive, language or motor composite scores in the SGA children when compared with the reference AGA children.

\section{Outcome in TGA infants}

Significantly lower composite scale scores were seen in cognitive $(p=0.036)$, language $(p=0.024)$ and motor $(p=0.051)$ domains in the TGA compared with AGA children. The Hedges' $g$ effect size was $0.375,0.35$ and 0.41 for cognitive, language and motor domains, respectively (table 2 ).

\section{Post hoc analysis}

To examine the effect of the overlap between the groups, we also measured the effect of being both SGA and TGA (STGA group). Infants in the STGA group had significantly lower cognitive and motor scores, with a trend towards decreased language scales. The median (IQR) cognitive score was 90 (85-95) compared with the reference AGA group median (IQR) of 95 
Table 2 BSIDIII composite scale scores across the developmental domains in each of the study groups

\begin{tabular}{|c|c|c|c|c|}
\hline Outcome & SGA $(n=51)$ & TGA $(n=51)$ & STGA $(n=13)$ & AGA $(n=189)$ \\
\hline Cognitive score & $95(90-100)$ & $95(90-97.5)$ & 90 (85-95) & $95(90-100)$ \\
\hline p Value* & 0.15 & 0.036 & 0.005 & - \\
\hline Hedges' $g$ effect size & 0.20 & 0.375 & 0.79 & - \\
\hline Language score & 109 (97-117) & 100 (94-109) & 99 (88-109) & $109(100-115)$ \\
\hline p Value & 0.57 & 0.024 & 0.129 & - \\
\hline Hedges' $g$ effect size & 0.06 & 0.35 & 0.57 & - \\
\hline Motor score & $103(94-110)$ & $100(91-97)$ & $96(90-103)$ & $103(97-110)$ \\
\hline$p$ Value & 0.95 & 0.051 & 0.043 & - \\
\hline Hedges' $g$ effect size & 0.08 & 0.41 & 0.71 & - \\
\hline Crude OR $(95 \% \mathrm{Cl})$ & 1.37 (0.62 to 3.00$)$ & 1.73 (0.8 to 3.72 ) & 4.58 (1.44 to 14.62$)$ & - \\
\hline p Value & 0.44 & 0.16 & 0.01 & \\
\hline Adjusted OR (95\% CI) & $1.36(0.60$ to 3.18$)$ & $1.78(0.80$ to 3.99$)$ & $5.00(1.46$ to 17.13$)$ & - \\
\hline p Value & 0.45 & 0.16 & 0.01 & \\
\hline Age at assessment & 26.65 (1.6) & $26.6(1.5)$ & $26.5(1.7)$ & $26.21(3.1)$ \\
\hline p Value & 0.16 & 0.21 & 0.54 & \\
\hline
\end{tabular}

Table 3 Child Behaviour Checklist parental report scales of behavioural difficulties at 24 months in each of the study groups

\begin{tabular}{|c|c|c|c|}
\hline Outcome & SGA & TGA & AGA \\
\hline Total problem score & $15.5(9-26.5)$ & $23.9(12-31)$ & $18(9-28)$ \\
\hline$p$ Value & 0.53 & 0.17 & - \\
\hline Internal raw score & $3(1-6)$ & $3.5(1-7)$ & $3(1-7)$ \\
\hline $\mathrm{p}$ Value & 0.58 & 0.82 & - \\
\hline External raw score & $7(2.75-11)$ & $7.5(4.75-14.0)$ & $8(4-11)$ \\
\hline$p$ Value & 0.78 & 0.03 & - \\
\hline ADH subscale & $2(1-5)$ & $4.0(1.75-5.25)$ & $3(1-4)$ \\
\hline \multicolumn{4}{|c|}{$\begin{array}{l}\text { Data are median (IQR). } \\
\text { Statistical significance set at } p<0.05 . p \text { Value denotes difference between study } \\
\text { groups and reference AGA group calculated by Mann-Whitney } U \text { test. } \\
\text { ADH, attention-deficit hyperactivity; AGA, appropriate-for-gestational age; } \\
\text { SGA, small-for-gestational age; TGA, thin-for-gestational age. }\end{array}$} \\
\hline
\end{tabular}

(90-100), $\mathrm{p}=0.005$. The effect size for cognitive outcome was a 0.71 SD reduction in cognitive score. Overall, the odds of developmental delay at 2 years was 5.01 (95\% CI 1.46 to 17.13 ), $\mathrm{p}=0.010$ compared with the AGA group following adjustment for sex, smoking status, SE index and maternal total years of schooling.

\section{Behavioural outcome}

The CBCL scores were compared in each of the groups with the AGA reference group. No significant difference was seen in parental report of behavioural difficulties in either study group compared with the reference AGA group, except for a small, but statistically significant increase in reported attention-deficit/ hyperactivity scores in the TGA group (table 3 ).

Overall, the percentage of CBCL scores in the clinical range was low, that is, $1.6 \%$ for TPS, $1.3 \%$ for IPS, $2.5 \%$ for EPS and $1.4 \%$ for the attention-deficit hyperactivity domain (ADHD). There was a trend towards increased rates in the TGA group (3.4\% TPS, 1.7\% IPS, 5.2\% EPS and 3.0\% ADHD subscale); however, this did not reach statistical significance.

\section{DISCUSSION}

We have shown that measurement of body composition may aid significantly in the retrospective identification of infants with poor developmental progress following suboptimal intrauterine growth. Classifying infants by birthweight centile, even centiles customised for maternal anthropometrics and ethnicity, did not allow us to reliably identify those most at risk retrospectively. Although SGA infants had a trend to lower BSIDIII scores, this was not significant. Neither was the OR that they would need intervention at 2 years. Categorising the cohort based on body fat composition at birth alone gave greater confidence, with significantly lower composite scale scores in all three domains in children with a body fat $<10$ th percentile at birth. This equates to approximately $<5 \%$ body fat at birth in term infants in our cohort. $^{21}$ The highest risk of poor outcome was seen in infants born STGA, who had a fivefold increased risk of an abnormal BSIDIII at 2 years.

The mean birth weight of the TGA infants was $3215 \mathrm{~g}$, lying on the 30th percentile line using customised birthweight centiles. Many (60/81 (74\%)) of these infants did not have a birth weight <10th centile and would therefore not be flagged as growth restricted, as their genetic growth potential may have been in the high normal range. Clinicians are familiar with $3.5 \mathrm{~kg}$ infants who are long, thin and asymmetrically grown, as they were destined to be $500-1000 \mathrm{~g}$ heavier. We have shown for the first time that these 'normal range' infants may also be at risk of long-term neurodevelopmental effects. Although the adjusted OR for developmental delay did not reach statistical significance in our population this is likely due to inadequate power, as the TGA group had significantly lower composite scores and a medium $(>0.3)$ effect size across all three measured domains, indicating a clinically significant effect.

The greatest effect on outcome was seen in the STGA group. Measuring body composition allowed us to identify those SGA infants with the most significant growth restriction. Assessing body composition in the first days after birth would allow clinicians to prospectively rule out those SGA infants who are 'small normal' with normal body composition, who have reached their 
genetic potential and are therefore low risk. ${ }^{29}$ In tandem, SGA infants with low \%BF could be offered paediatric follow-up and developmental monitoring as they are at high risk of later difficulties. There is increasing evidence that targeted intervention and environmental enrichment may improve later executive function, particularly at young ages and in socially disadvantaged areas. ${ }^{30}$

No internationally recognised clinical definition for FGR exists. The diagnosis of FGR is complex and requires serial noninvasive measurements of fetal growth, using both ultrasound estimations of growth parameters and Doppler examination of blood flow through the umbilical and fetal vessels. ${ }^{41}$ Although giving excellent information in expert hands, ultrasound has accepted limitations including subjectivity, an acknowledged margin of error and the requirement for specialised equipment and operator skill sets, which limits availability in low resource settings. Therefore, many term infants with FGR may go undiagnosed. ${ }^{31}$ Assessing whether the infant is FGR or 'small normal' in the postnatal period is also fraught with difficulty. Skinfold thickness measurement is prone to large intraobserver variability and has not been previously linked with poor developmental outcome. ${ }^{32}$ Direct body fat estimation may allow improved confirmation of suboptimal growth. These difficulties have led to inconsistent findings, with some studies showing a modest or negligible effect. ${ }^{18}$

We are aware that we have used the same birth cohort to determine both the normative reference ranges and the effect of being at the lowest end of the \%BF distribution at birth. Our cut-offs may not be applicable to other, more heterogeneous populations. Our cohort was low risk, primiparous mothers of European origin. The risk factors for SGA in our maternal cohort have been previously described. ${ }^{33}$ Less than $5 \%$ of our mothers were non-Caucasian and the majority had a tertiary level education. However, information on normal body composition at birth is increasing and our reference ranges for $\% \mathrm{BF}$ at term are similar to that reported in Australian, French and American neonatal cohorts. ${ }^{34} 35$

We did not find any significant behavioural difficulties in our cohort; however, they were assessed at an age where difficulties may not be apparent. In addition, we relied on parental report as direct behavioural measures are difficult at this age. Previous work has shown significant increases in the areas of attention and hyperactivity, which seems to be linear in effect. ${ }^{6}$ We hope to examine this question again at school age through repeated behavioural measures. Further limitations include the small sample size resulting in wide CIs, residual confounding in observational research and post hoc analyses, which may introduce bias to the study findings, as well as to unmeasured potential confounders.

The strengths of our study include its prospective design providing detailed information on maternal characteristics including SE strata, occupation and smoking status. The exposed infants (SGA, TGA) and reference (unexposed) infants were also selected based on strict criteria including body fat estimation and were followed up using standardised developmental assessment tools conducted by a sole research psychologist blinded to the neonatal status of the infants. We did not examine antenatal assessments of growth as we wished to assess the ability of postnatal body composition assessment alone to aid paediatric assessment of risk. Inclusion of antenatal concerns and ultrasound estimation of fetal growth and Doppler pulsatility indices may allow a more detailed risk assessment to take place. This study has examined the place of neonatal body composition in postnatal risk assessment.

\section{Conclusion}

We have shown for the first time that body composition at birth may be an important indicator of risk in infants with inadequate fetal growth. TGA infants had global developmental delay at 2 years compared with their appropriately grown counterparts, and more significant than that seen in SGA infants. The greatest risk for developmental delay was seen in STGA children.

\section{Author affiliations}

${ }^{1}$ Irish Centre for Fetal and Neonatal Translational Research, Cork University Maternity Hospital, University College Cork, Cork, Ireland

${ }^{2}$ Paediatrics \& Child Health, University College Cork, Cork, Ireland

${ }^{3}$ Department of Epidemiology and Public Health, Western Gateway Building,

University College Cork, Cork, Ireland

${ }^{4}$ Department of Obstetrics and Gynaecology, Cork University Maternity Hospital, Cork, Ireland

${ }^{5}$ Cork Centre for Vitamin D and Nutrition Research, School of Food and Nutritional Sciences, University College, Cork, Ireland

\section{Twitter Follow Sinead O'Neill at @sineadoneill13}

Acknowledgements The authors thank the pregnant women who participated in the SCOPE study and the SCOPE Ireland research team. They gratefully acknowledge those who helped in the gathering these data including all BASELINE study employees, both past and present. In particular, they also thank all the parents and children of the BASELINE study for their time, effort, and enthusiasm in attending multiple appointments in the busy first years of life.

Contributors SMO completed analysis of the data and drafted the first and subsequent drafts of the manuscript. GH was responsible for the neurodevelopmental assessments, data reporting and editing of the final manuscript. ASK provided statistical input into the study design, planned and supervised the data analysis and planned and edited the final manuscript. JOBH aided in study design, governance of the BASELINE Birth Cohort Study and contributed to the final manuscript. LCK is the principal investigator of the SCOPE pregnancy study, and co-investigator of the BASELINE Birth Cohort. LCK assisted in the study design and manuscript preparation. MK aided in study design, governance of the BASELINE Birth Cohort Study and contributed and edited the final manuscript. DMM is the principal investigator of the BASELINE Birth Cohort Study and planned the study design and governed its execution. DMM aided in data analysis, manuscript planning, preparation and final editing.

Funding The Cork BASELINE Birth Cohort Study was funded by the National Children's Research Centre and the UK Food Standards Agency (FSA T07060). GH was partly funded by an educational grant from Danone Nutricia. The Irish Centre for Fetal and Neonatal Translational Research is a Science Foundation Ireland funded Centre (12/RC/2272). The SCOPE study was funded by the Health Research Board, Ireland (CSA/2007/2).

Competing interests JOBH has received separate funding from Danone Nutricia. Ethics approval Cork Research Ethics Committee.

Provenance and peer review Not commissioned; externally peer reviewed.

Data sharing statement Data from the Cork BASELINE Birth Cohort Study can be made available to researchers following application to the research committee with a completed research application form. These are available on request through our research team. Contact details and further information are available on www. baselinestudy.net.

\section{REFERENCES}

1 Savchev S, Sanz-Cortes M, Cruz-Martinez R, et al. Neurodevelopmental outcome of full-term small-for-gestational-age infants with normal placental function. Ultrasound Obstet Gynecol 2013;42:201-6.

2 Mwaniki MK, Atieno M, Lawn JE, et al. Long-term neurodevelopmental outcomes after intrauterine and neonatal insults: a systematic review. Lancet 2012;379:445-52.

3 Barker DJ, Eriksson JG, Forsen T, et al. Fetal origins of adult disease: strength of effects and biological basis. Int J Epidemiol 2002;31:1235-9.

4 Resnik R. Intrauterine growth restriction. Obstet Gynecol 2002;99:490-6.

5 Figueras F, Gardosi J. Intrauterine growth restriction: new concepts in antenatal surveillance, diagnosis, and management. Am J Obstet Gynecol 2011;204:288-300.

6 Miller SL, Huppi PS, Mallard C. The consequences of fetal growth restriction on brain structure and neurodevelopmental outcome. J Physiol (Lond) 2016;594:807.

7 Kingdom J, Smith G, Baker P. Diagnosis and management of IUGR. In: Kingdom J, Baker $\mathrm{P}$, eds. Intrauterine growth restriction aetiology and management. London: Springer, 2000 
8 Benavides-Serralde A, Hernandez-Andrade E, Fernandez-Lara A, et al. Accuracy of different equations for estimating fetal weight. Gynecol Obstet Invest 2011;72:264-8.

9 Mayer $C$, Joseph $\mathrm{K}$. Fetal growth: a review of terms, concepts and issues relevant to obstetrics. Ultrasound Obstet Gynecol 2013;41:136-45.

10 Arcangeli T, Thilaganathan B, Hooper $\mathrm{R}$, et al. Neurodevelopmental delay in small babies at term: a systematic review. Ultrasound Obstet Gynecol 2012;40:267-75.

11 Geva R, Eshel R, Leitner $Y$, et al. Neuropsychological outcome of children with intrauterine growth restriction: a 9-year prospective study. Pediatrics 2006:118:91-100.

12 von Beckerath A-K, Kollmann M, Rotky-Fast C, et al. Perinatal complications and long-term neurodevelopmental outcome of infants with intrauterine growth restriction. Am J Obstet Gynecol 2013;208:130. e131-130. e136.

13 De Jesus LC, Pappas A, Shankaran S, et al. Outcomes of small for gestational age infants born at $<27$ weeks' gestation. J Pediatr 2013;163:55-60. e53.

14 Parra-Saavedra M, Crovetto F, Triunfo S, et al. Neurodevelopmental outcomes of near-term small-for-gestational-age infants with and without signs of placental underperfusion. Placenta 2014;35:269-74.

15 Serenius F, Sjörs $G$, Blennow $M$, et al. EXPRESS study shows significant regional differences in 1-year outcome of extremely preterm infants in Sweden. Acta Paediatr 2014;103:27-37

16 McCowan LM, Harding JE, Stewart AW. Customised birthweight centiles predict SGA pregnancies with perinatal morbidity. BJOG 2005;112:1026-33.

17 Zhang $\mathrm{X}$, Cnattingius S, Platt RW, et al. Are babies born to short, primiparous, or thin mothers "normally" or "abnormally" small? J Pediatr 2007;150:603-7. e603.

18 Alkandari F, Ellahi A, Aucott L, et al. Fetal ultrasound measurements and associations with postnatal outcomes in infancy and childhood: a systematic review of an emerging literature. J Epidemio/ Community Health 2015:69:41-8.

19 Gardosi J, Mul T, Mongelli M, et al. Analysis of birthweight and gestational age in anteparturn stillbirths. BJOG 1998:105:524-30.

20 Gardosi J, Figueras F, Clausson B, et al. The customised growth potential: an international research tool to study the epidemiology of fetal growth. Paediatr Perinat Epidemiol 2011:25:2-10.

21 Hawkes CP, Hourihane JOB Kenny LC, et al. Gender-and gestational age-specific body fat percentage at birth. Pediatrics 2011;128:e645-51.
22 O'Donovan SM, Murray DM, Hourihane JOB, et al. Cohort profile: the Cork BASELINE Birth Cohort Study: babies after SCOPE: evaluating the longitudinal impact on neurological and nutritional endpoints. Int J Epidemiol 2015;44:764-75.

23 North RA, McCowan LM, Dekker GA, et al. Clinical risk prediction for pre-eclampsia in nulliparous women: development of model in international prospective cohort. BMJ 2011:342:d1875.

24 Bayley N. Bayley scales of infant and toddler development: Bayley-III. Harcourt Assessment, Psychological Corporation, 2006

25 Johnson S, Moore T, Marlow N. Using the Bayley-III to assess neurodevelopmental delay: which cut-off should be used? 2014;75:670-4

26 Achenbach TM, Ruffle TM. The Child Behavior Checklist and related forms for assessing behavioral/emotional problems and competencies. Pediatr Rev 2000;21:265-71.

27 Rosenthal R, Cooper H, Hedges L. Parametric measures of effect size. The handbook of research synthesis. 1994:231-44.

28 Davis P, McLeod K, Ransom M, et al. The New Zealand socioeconomic index of occupational status (NZSEI). Wellington: Statistics New Zealand, 1997.

29 Zhang X, Cnattingius S, Platt RW, et al. Are babies born to short, primiparous, or thin mothers "normally" or "abnormally" small? J Pediatr 2007;150:603-7, 607 e601-603.

30 Diamond A, Ling DS. Conclusions about interventions, programs, and approaches for improving executive functions that appear justified and those that, despite much hype, do not. Dev Cogn Neurosci 2016;18:34-48

31 Alberry M, Soothill P. Management of fetal growth restriction. Arch Dis Child Fetal Neonatal Ed 2007;92:F62-67.

32 Ulijaszek SJ, Kerr DA. Anthropometric measurement error and the assessment of nutritional status. Br J Nutr 1999;82:165-77.

33 McCowan LM, Thompson JM, Taylor RS, et al. Clinical prediction in early pregnancy of infants small for gestational age by customised birthweight centiles: findings from a healthy nulliparous cohort. PLOS ONE 2013;8:e70917.

34 Deierlein $\mathrm{AL}$, Thornton J, Hull $\mathrm{H}$, et al. An anthropometric model to estimate neonatal fat mass using air displacement plethysmography. Nutr Metab (Lond) 2012:9:21.

35 Au CP, Raynes-Greenow CH, Turner RM, et al. Fetal and maternal factors associated with neonatal adiposity as measured by air displacement plethysmography: a large cross-sectional study. Early Hum Dev 2013;89:839-43. 